\title{
Polygonal Approximation Using an Artificial Bee Colony Algorithm
}

\author{
Shu-Chien Huang \\ Department of Computer Science, National Pingtung University, Pingtung City 90003, Taiwan \\ Correspondence should be addressed to Shu-Chien Huang; schuang@mail.nptu.edu.tw
}

Received 8 August 2014; Revised 11 December 2014; Accepted 15 December 2014

Academic Editor: Debasish Roy

Copyright ( 2015 Shu-Chien Huang. This is an open access article distributed under the Creative Commons Attribution License, which permits unrestricted use, distribution, and reproduction in any medium, provided the original work is properly cited.

\begin{abstract}
A polygonal approximation method based on the new artificial bee colony (NABC) algorithm is proposed in this paper. In the present method, a solution is represented by a vector, and the objective function is defined as the integral square error between the given curve and its corresponding polygon. The search process, including the employed bee stage, the onlooker bee stage, and the scout bee stage, has been constructed for this specific problem. Most experiments show that the present method when compared with the DE-based method can obtain superior approximation results with less error norm with respect to the original curves.
\end{abstract}

\section{Introduction}

Polygonal approximation is a common representation of digital curves [1]. A polygonal representation of a digital curve can significantly reduce the amount of data needed to be processed while at the same time preserving important information about the curve. It can be applied in shape analysis, pattern classification, image understanding, 3D reconstruction, and CAD applications. In recent decades, a number of methods have been proposed for approximation. In general, they can be categorized into three approaches: (1) given the number of breakpoints, $m$, find the approximation with $m$ breakpoints such that the approximation error is minimized; (2) given an error $\varepsilon$, find the approximation with the minimum number of breakpoints that is distant from the given curve by no more than $\varepsilon$; and (3) dominant pointdetection approaches. In this paper, the present method is categorized into the first approach for polygonal approximation.

Teh and Chin [2] indicated that dominant point detection relies on the determination of a local support region rather than the estimation of discrete curvature. B. K. Ray and K. S. Ray [3] developed a technique to determine the polygon of an object curve by simultaneously maximizing the line length and minimizing the approximation error. Pei and Horng [4] proposed a dynamic programming technique for constructing an approximation using circular arcs. Huang and Sun
[5] developed a method based on genetic algorithms for the polygonal approximation of plane curves. Ho and Chen [6] solved the polygonal approximation problem using an efficient evolutionary algorithm with a novel orthogonal array crossover, which is an efficient general-purpose algorithm capable of solving large parameter optimization problems. Yin [7] proposed a polygonal approximation method using an ant colony search algorithm. Sarkar [8] formulated the polygonal approximation task as a minimization problem with ISE as the objective function and then used the differential evolution-based method to solve it. Kolesnikov [9] presented a method based on searching for the shortest path in a feasibility graph for the polygonal approximation of digital curves with a minimum number of approximation segments for a given error bound. Wang et al. [10] proposed an approach, integer particle swarm optimization, for polygonal approximation. Their method found a polygon with the minimum number of vertices whose integral square error is less than a given maximum tolerance.

In recent years, researchers have attempted to apply nature-inspired methods to the problem of pattern recognition and image processing. In this paper, the new artificial bee colony (NABC) algorithm [11] is employed for approximation of digital curves with line segments. The present method can output solutions very close to the optimal one in a relatively short time. The remainder of this paper is organized as 
follows. In Section 2, the problem is illustrated. In Section 3, the NABC algorithm is described. In Section 4, the NABC algorithm for polygonal approximation is presented. In Section 5, the experimental studies for the comparison of the present method with the DE-based method are shown. Finally, conclusions are given in Section 6.

\section{Problem Statement}

Given a curve with $n$ clockwise-ordered points, denoting $C=$ $\left\{p_{0}, p_{1}, p_{2}, \ldots, p_{n-1}\right\}$, let $\widetilde{p_{x} p_{y}}$ and $\overline{p_{x} p_{y}}$ represent the arc and the chord starting at point $p_{x}$ and continuing to point $p_{y}$ in the clockwise direction along the original curve $C$. The approximation error of line segment $\overline{p_{x} p_{y}}$, denoted as $e_{x, y}$, is defined as

$$
e_{x, y}=\sum d^{2}\left(p_{i}, \overline{p_{x} p_{y}}\right), \quad \forall p_{i} \in \widetilde{p_{x} p_{y}}
$$

where $d\left(p_{i}, \overline{p_{x} p_{y}}\right)$ is the perpendicular distance of arc point $p_{i}$ to the chord $\overline{p_{x} p_{y}}$.

The objective of the polygonal approximation problem is to find a polygon, assuming that the set of its breakpoints is $C^{*}=\left\{p_{a(1)}, p_{a(2)}, \ldots, p_{a(m)}\right\}$, where $0 \leq a(1)<a(2)<\cdots<$ $a(m) \leq n-1$, such that the integral square error between the polygon and the given curve is minimized. The integral square error (ISE) is defined as follows:

$$
\mathrm{ISE}=e_{a(1), a(2)}+e_{a(2), a(3)}+\cdots+e_{a(m-1), a(m)}+e_{a(m), a(1)} .
$$

Therefore, there exists $C_{(n, m)}(=n ! /[m !(n-m) !])$ combinations to consider when searching for the $m$ breakpoints of the given curve. Generally, this problem is not trivial and always turns into a time-consuming iterative search.

\section{The New Artificial Bee Colony (NABC) Algorithm}

The artificial bee colony (ABC) algorithm [12-14] was proposed by Karaboga for optimizing numerical problems. Karaboga and Ozturk [15] applied it to training feed-forward neural networks to classify nine data sets. Xu and Duan [16] proposed an artificial bee colony algorithm with edge potential function to accomplish the target recognition task for aircraft. Zhang et al. [17] used the ABC algorithm for clustering. Zhu and Kwong [18] proposed the Gbestguided $\mathrm{ABC}$ (GABC) algorithm, which takes advantage of the information of the global best solution to guide the search for new candidate solutions in order to improve the exploitation. A set of numerical benchmark functions were tested, and the results show that the GABC algorithm can outperform the $\mathrm{ABC}$ algorithm in most of their experiments. Pan et al. [19] proposed a discrete artificial bee colony (DABC) algorithm to solve the lot-streaming flow shop scheduling problem. Horng [20] proposed the multilevel thresholding algorithm for image segmentation based on the technology of the artificial bee colony algorithm, while Gao et al. [21] proposed a modified ABC algorithm, which is based on each bee searching only around the best solution of the previous iteration in order to improve the exploitation. Draa and
Bouaziz [22] used an artificial bee colony algorithm for image contrast enhancement. In their method, the grey levels of the input image are replaced by a new set of grey levels. Talatahari et al. [23] presented a new optimization method based on the artificial bee colony algorithm for solving parameter identification problems. Sarkar et al. [24] presented a perturbed martingale approach to global optimization, and the results show that their method appears to substantively improve upon the performance of the particle swarm optimization method.

The ABC algorithm simulates the intelligent foraging behavior of honey bee swarms. The colony of artificial bees contains three groups of bees: employed bees, onlooker bees, and scout bees. In the ABC algorithm, the first half of the colony consists of employed bees while the second half is constituted of the onlooker bees. A bee associated with a food resource is called an employed bee, while a bee waiting in the dance area to get information concerning food sources is called an onlooker bee. An employed bee whose food source is exhausted by the employed and onlooker bees becomes a scout. The scout bee carries out random searches to discover new sources. The number of food sources, denoted by SN, is equal to the number of employed bees. The position of a food source represents a possible solution to the optimization problem, while the nectar amount of a food source corresponds to the quality (fitness) of the associated solution. Each solution $x_{i}(i=1,2, \ldots, \mathrm{SN})$ is an $m$-dimensional vector. Here, $m$ is the number of optimization parameters and is also the number of breakpoints of a polygon in this paper.

In [11], an improved version of ABC, called NABC, was proposed. A set of twelve benchmark functions were used in their experiments. The simulation results showed that the NABC algorithm is significantly better or at least comparable to the original $\mathrm{ABC}$ algorithm. In the initialization stage, NABC generates a randomly distributed initial population of SN solutions (food source positions). The fitness of all solutions is evaluated. Then, a solution pool is constructed by storing the best $100 p \%$ solutions in the current swarm with $p \in(0,1]$.

In the employed bee stage, the original $\mathrm{ABC}$ algorithm generates a candidate solution $v_{i}$ in the neighborhood of its present solution $x_{i}$ as follows:

$$
v_{i, j}=x_{i, j}+\phi_{i j}\left(x_{i, j}-x_{k, j}\right),
$$

where $k$ is a randomly produced index which is different from $i, j$ is a random dimension index selected from the set $\{1,2, \ldots, m\}$, and $\phi_{i j}$ is a random number in the range $[-1,1]$. In [11], the new ABC/best/1 strategy was proposed in the employed bee stage. The NABC algorithm generates a candidate solution $v_{i}$ as follows:

$$
v_{i, j}=x_{\text {best }, j}^{p}+\phi_{i j}\left(x_{r 1, j}-x_{r 2, j}\right),
$$

where $x_{\text {best }, j}^{p}$ is randomly chosen from the solution pool and $x_{r 1}, x_{r 2}$ are two randomly selected solutions from the current swarm with $i \neq r 1 \neq r 2$.

In the onlooker bee stage, an artificial onlooker bee chooses a food source depending on the probability value 
(1) // Initialization stage

Generate the initial population $x_{i}, i=1, \ldots, S N$

trial $_{i}=0, i=1, \ldots, S N$

set cycle to 1

(2) Evaluate the fitness $f i t\left(x_{i}\right)$ of the population

Repeat

(3) Update the solution pool

(4) // Employed bee stage

For each employed bee

Produce new solution (food source position) $v_{i}$ by using (4)

Calculate the fitness value $f i t\left(v_{i}\right)$

If $f i t\left(v_{i}\right)>f i t\left(x_{i}\right)$ then $x_{i}$ is replaced by $v_{i}$ and trial $_{i}$ is set to 0 else trial $_{i}=$ trial $_{i}+1$ End

(5) Calculate the probability values prob by using (5) for the solutions $x_{i}(i=1, \ldots, S N)$

(6) // Onlooker bee stage

For each onlooker bee

Select a solution $x_{i}$ depending on prob $_{i}$

Produce new solution (food source position) $v_{i}$ by using (4)

Calculate the fitness value $f i t\left(v_{i}\right)$

If $f i t\left(v_{i}\right)>f i t\left(x_{i}\right)$ then $x_{i}$ is replaced by $v_{i}$ and trial $_{i}$ is set to 0 else trial $_{i}=$ trial $_{i}+1$ End

(7) Memorize the best solution so far

(8) // Scout bee stage

If there is an abandoned solution ( trial $_{i}>$ limit) for the scout then replace it with a new solution which will be randomly produced cycle $=$ cycle +1

Until cycle $\leqq M C N$

Algorithm 1

associated with that food source, prob $_{i}$, calculated by the following expression:

$$
\begin{aligned}
\operatorname{prob}_{i}= & 0.9 \times \frac{\text { fit }\left(x_{i}\right)}{\max \left\{\text { fit }\left(x_{1}\right), \text { fit }\left(x_{2}\right), \ldots, \text { fit }\left(x_{\mathrm{SN}}\right)\right\}} \\
& +0.1,
\end{aligned}
$$

where fit $\left(x_{i}\right)$ is the fitness value of the solution $x_{i}$. In the scout bee stage, if a solution $x_{i}$ is abandoned, then the scout bee discovers a new food source to replace $x_{i}$.

The difference between the NABC algorithm and the original $\mathrm{ABC}$ algorithm is that the NABC algorithm constructs a solution pool and a candidate solution $v_{i}$ is generated by using (4) instead of (3). In the NABC approach, bees can search the neighborhood of different best solutions. This can help avoid fast attraction and is helpful for accelerating the convergence speed.

The flowchart of the NABC algorithm is given in Figure 1. The pseudocode of the NABC algorithm is shown in Algorithm 1.

\section{The Proposed NABC Algorithm for Polygonal Approximation}

In this section, the representation of solutions and the fitness function are described, and the search process of the NABC algorithm for accomplishing the task of polygonal approximation is also presented.

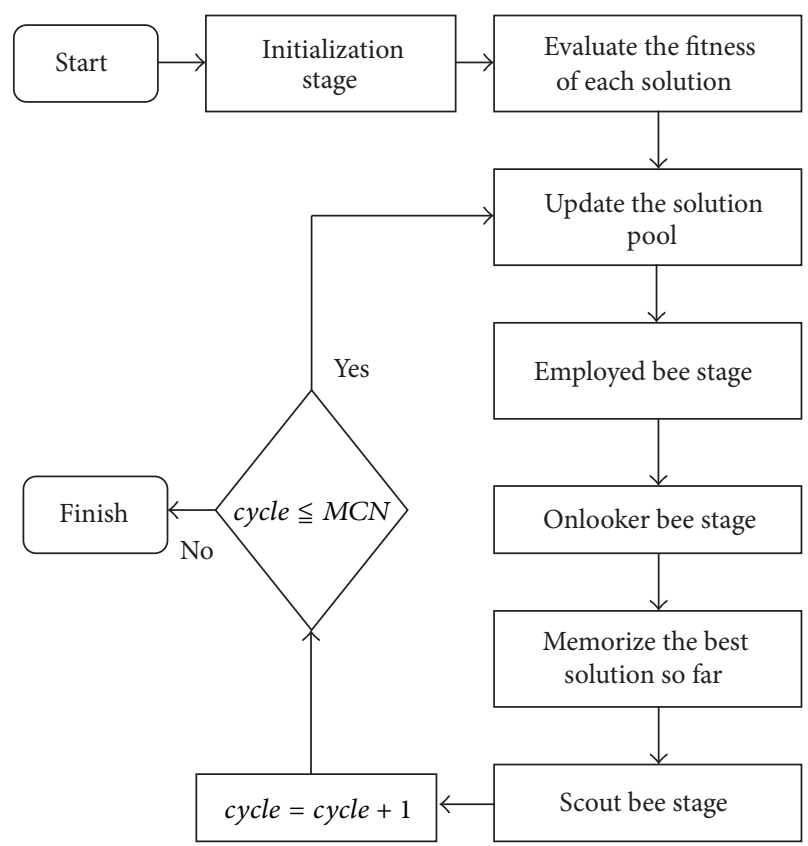

FIGURE 1: Flow chart of the NABC algorithm.

4.1. Representation of Solutions. In the initialization stage, the SN solution $x_{i}(i=1,2, \ldots, \mathrm{SN})$ is generated, where $x_{i}=\left(x_{i, 1}, x_{i, 2}, \ldots, x_{i, m}\right), x_{i, j}$ is the real number, and the 


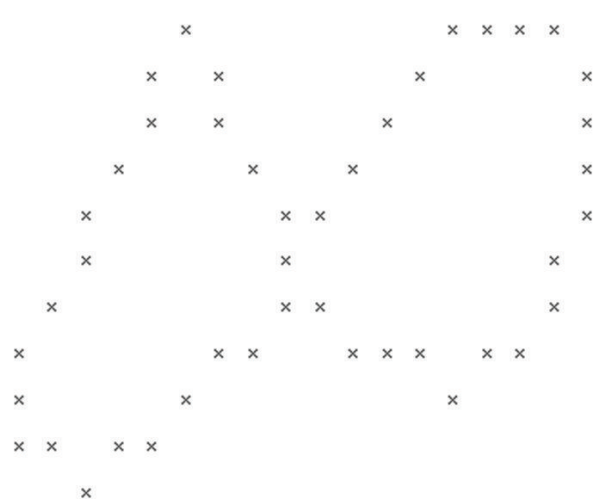

(a) The point number $=45$

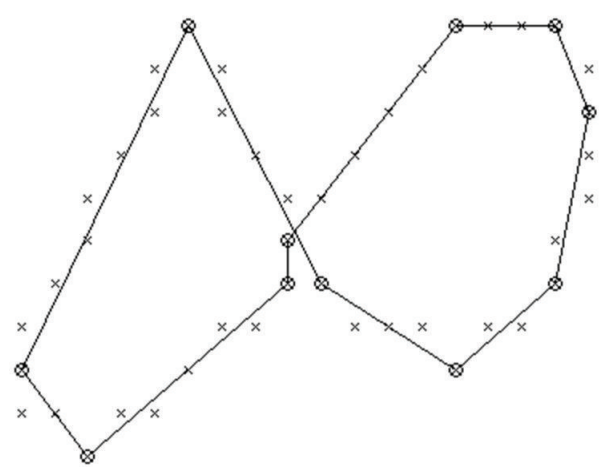

(c) Number of breakpoints $=11$

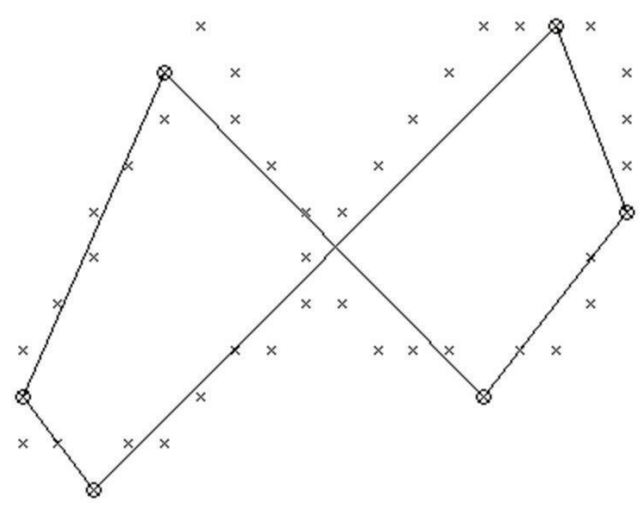

(b) Number of breakpoints $=6$

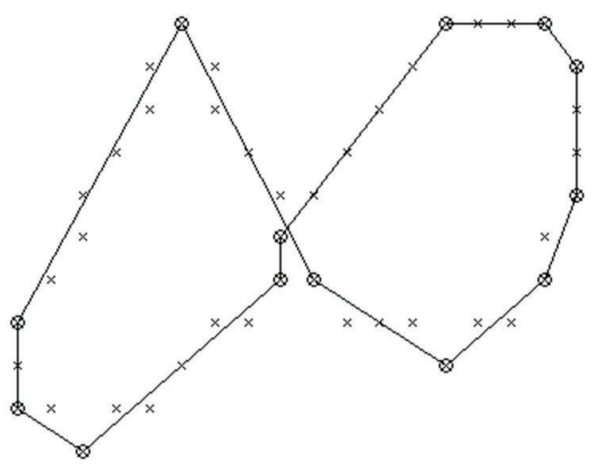

(d) Number of breakpoints $=13$

FIGURE 2: The approximated polygons for the figure-of-eight curve obtained by the present method. (a) Original curve and (b)-(d) results using the present method.

$0 \leq x_{i, j}<x_{i, j+1}<n$ for all $j$. Then, the value of cycle is set to 0 and the trial number of each solution $x_{i}$, trial $_{i}$, is equal to 0 .

4.2. Fitness Function. The solution $x_{i}=\left(x_{i, 1}, x_{i, 2}, \ldots, x_{i, m}\right)$ corresponds to a polygon and the set of its breakpoints is $\left\{p_{a(1)}, p_{a(2)}, \ldots, p_{a(m-1)}, p_{a(m)}\right\}$, where $a(j)=$ floor $\left(x_{i, j}\right), j \in$ $\{1,2, \ldots, m\}$. First, the integral square error (ISE) is computed by using (2). Then, the fitness value can be calculated by the following expression:

$$
\text { fit }\left(x_{i}\right)=\frac{1}{(\operatorname{ISE}+1)} .
$$

\subsection{The Search Process}

4.3.1. The Employed Bee Stage. In this stage, the $i$ th $(i=1$, $2, \ldots, \mathrm{SN}$ ) employed bee produces a new solution $v_{i}$ by using (4) and computes the fitness value of the new solution. To ensure that $v_{i, j}<v_{i, j+1}$, the elements of each solution vector are sorted in ascending order of magnitude. If the fitness of the new solution $v_{i}$ is higher than that of the old solution $x_{i}$, the solution $x_{i}$ is replaced by $v_{i}$ and trial $i$ is set to 0 ; otherwise, the old solution $x_{i}$ is kept and trial ${ }_{i}$ is increased by 1 .

4.3.2. The Onlooker Bee Stage. First, the probability value prob $_{i}$ is calculated by using (5) for the solution $x_{i}$. Each onlooker bee which selects a solution $x_{i}$ depending on prob ${ }_{i}$ also produces a new solution $v_{i}$ by using (4). Then, it applies the greedy selection process between $v_{i}$ and $x_{i}$. As described in Section 4.3.1, if the fitness of the new solution $v_{i}$ is higher than that of the old solution $x_{i}$, the solution $x_{i}$ is replaced by $v_{i}$ and trial ${ }_{i}$ is set to 0 ; otherwise, the old solution $x_{i}$ is kept and trial $_{i}$ is increased by 1 .

4.3.3. The Scout Bee Stage. As described above, if the solution $x_{i}$ is not improved through the employed stage and the onlooker bee stage, the trial ${ }_{i}$ value of solution $x_{i}$ will be increased by 1 . If the trial ${ }_{i}$ of solution $x_{i}$ is more than the parameter "limit," the solution $x_{i}$ is considered to be an abandoned solution; meanwhile, the employed bee will be changed into a scout. The scout randomly produces the new solution to replace $x_{i}$. Then, the value of trial $i$ is set 0 , and this scout is changed into an employed bee.

4.3.4. Check the Termination Criterion. If the cycle is greater than the maximum cycle number $(\mathrm{MCN})$, then the algorithm is finished and the best solution found so far is output.

\section{Experimental Results}

The present method is experimentally tested and compared with the DE-based method [8]. The program is coded in C language and run on a PC with a Pentium $4 \mathrm{CPU}$. The control 


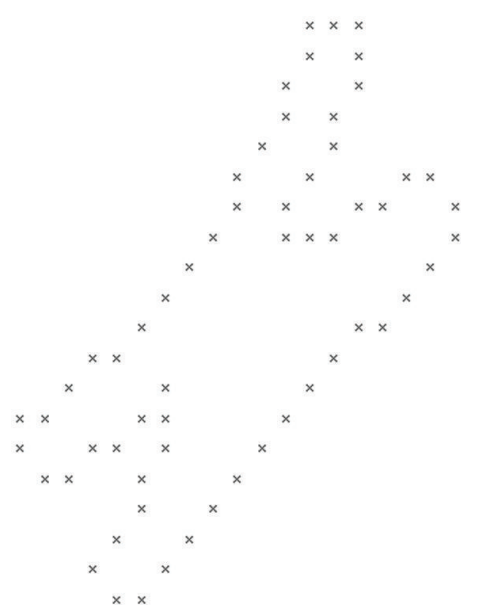

(a) The point number $=60$

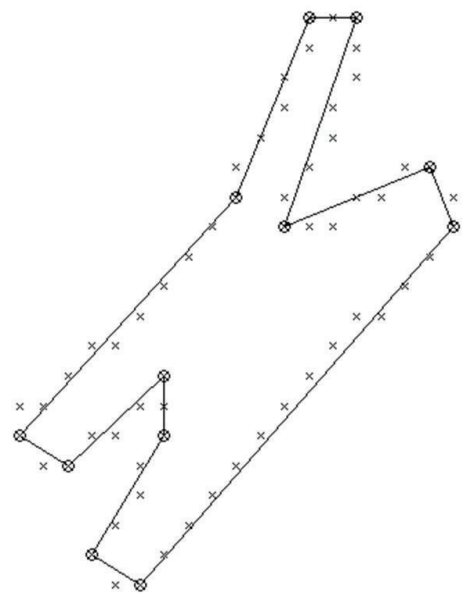

(c) Number of breakpoints $=12$

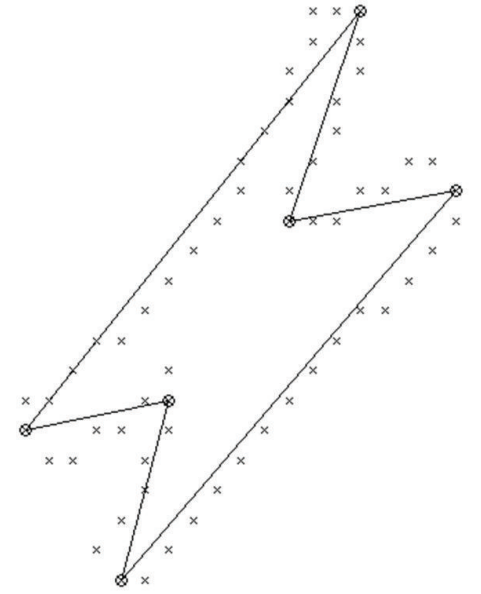

(b) Number of breakpoints $=6$

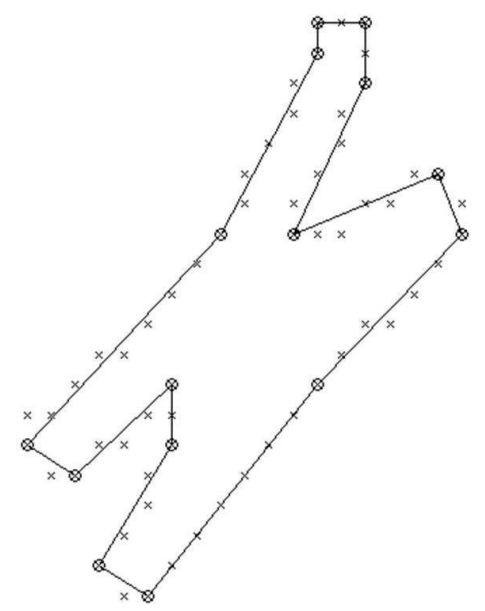

(d) Number of breakpoints $=15$

FIGURE 3: The approximated polygons for the chromosome-shaped curve obtained by the present method. (a) Original curve and (b)-(d) results using the present method.

parameter that is the number of food sources, which is equal to the number of employed bees or onlooker bees $(\mathrm{SN})$, is set to 100 , the value of "limit" is set to 50 , the maximum cycle number (MCN) is set to 600, and the parameter $p$ for constructing a solution pool is set to 0.06 in the experiments.

The DE algorithm is an evolutionary algorithm for solving numerical problems. The NABC algorithm is a novel swarm intelligent algorithm inspired by the foraging behaviors of a honeybee colony. The two methods have attracted the attention of researchers and have been widely used in solving engineering optimization problems. The main difference between the two methods is that they adopt different strategies to produce new solutions. The two methods start with an initial population of SN solutions. In the DE algorithm, new solutions are generated through the operations of mutation, crossover, and selection. In the NABC algorithm, as shown in Figure 1, new solutions are generated after performing a solution pool construction, employed bee stage, onlooker bee stage, and scout bee stage.

In order to verify the performance of the present method, four digital curves, namely, a figure-of-eight curve (see
Figure 2(a)), a chromosome-shaped curve (see Figure 3(a)), a leaf-shaped curve (see Figure 4(a)), and a semicircleshaped curve (see Figure 5(a)), are applied to be tested. The simulation conducted twenty independent runs. The best solutions, average solutions, and the standard deviations (S.D.) of the solutions are reported. The simulation results for the figure-of-eight curve, the chromosome-shaped curve, the leaf-shaped curve, and the semicircle-shaped curve are listed in Tables 1, 2, 3, and 4, respectively. For the average solutions, the present method outperforms the DE-based method. For the best solutions, it is shown that the present method produces better polygonal approximations than the DE-based method in most cases. In addition, the low S.D. reveals that the present method has higher stability than the DE-based method.

In Figures 2-5, Parts (b)-(d) show the best results obtained by the present method with different numbers of breakpoints employed. The computation times for the four digital curves are listed in Table 5. The present method can output solutions immediately because the processing time of all cases is less than 1 second in the experiments. 


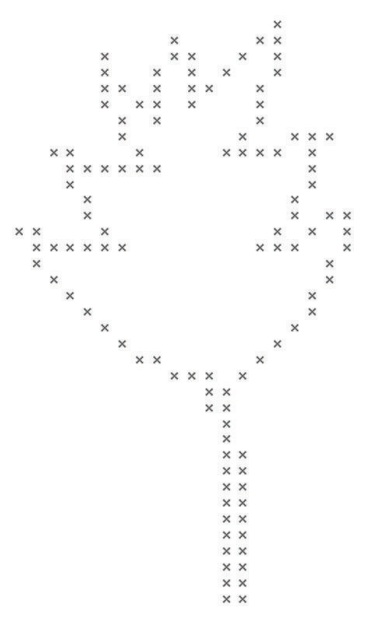

(a) The point number $=120$

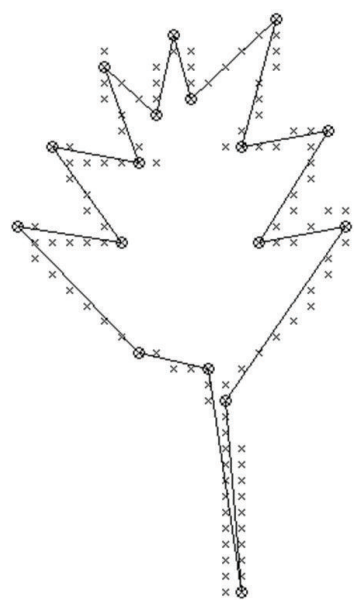

(c) Number of breakpoints $=17$

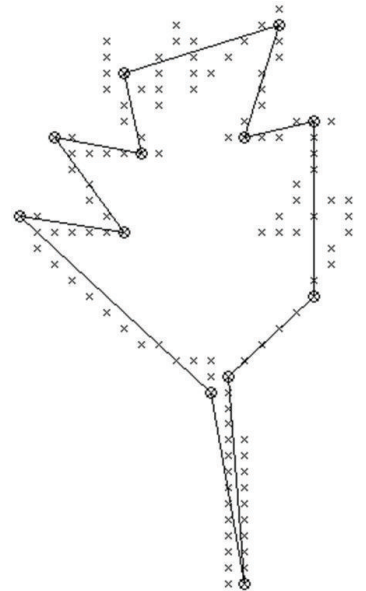

(b) Number of breakpoints $=12$

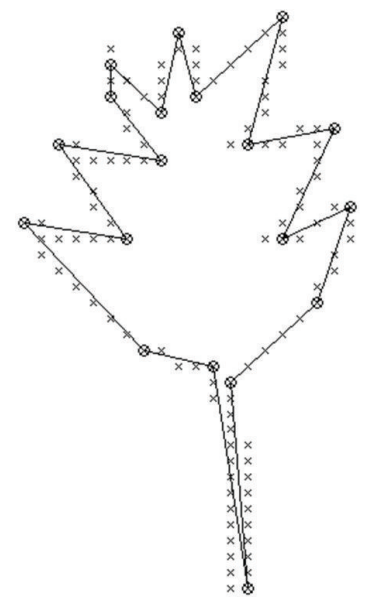

(d) Number of breakpoints $=19$

FIGURE 4: The approximated polygons for the leaf-shaped curve obtained by the present method. (a) Original curve and (b)-(d) results using the present method.

TABLE 1: Experimental results for the figure-of-eight curve.

\begin{tabular}{|c|c|c|c|c|c|c|}
\hline \multirow{2}{*}{ Number of breakpoints } & \multicolumn{3}{|c|}{ DE-based method [8] } & \multicolumn{3}{|c|}{ Present method } \\
\hline & ISE (best) & ISE (average) & S.D. & ISE (best) & ISE (average) & S.D. \\
\hline 6 & 17.494 & 17.494 & 0.000 & 17.494 & 17.494 & 0.000 \\
\hline 11 & 2.869 & 3.235 & 0.240 & 2.896 & 2.896 & 0.000 \\
\hline 13 & 2.042 & 2.509 & 0.277 & 2.042 & 2.042 & 0.000 \\
\hline
\end{tabular}

To demonstrate the applicability of the present method, it is applied to the screwdriver-shaped curve (see Figure 6(a)) and the fish-shaped curve (see Figure 7(a)). The maximum cycle number (MCN) is set to 1,000 for these two relatively larger curves. For the screwdriver-shaped curve, the point number of this curve is 267. Figures 6(b) and 6(c) show the best approximated polygon obtained through the present method with breakpoint numbers of 40 and 45, respectively. The computation times for Figures 6(b) and 6(c) are $2.547 \mathrm{~s}$ and $2.656 \mathrm{~s}$, respectively. For the fish-shaped curve, the point number is 537. Figures $7(\mathrm{~b})$ and 7 (c) show the best approximated polygon obtained through the present method with breakpoint numbers of 50 and 60, respectively. The computation times for Figures 7(b) and 7(c) are 4.671 s and $4.891 \mathrm{~s}$, respectively. The results of the approximation are tabulated in Table 6. It is seen that the present method can also give good approximation results.

\section{Conclusions}

A new approach based on the new artificial bee colony (NABC) algorithm has been developed for approximation of digital curves with line segments. Given the number of breakpoints, the algorithm finds an approximation with the 


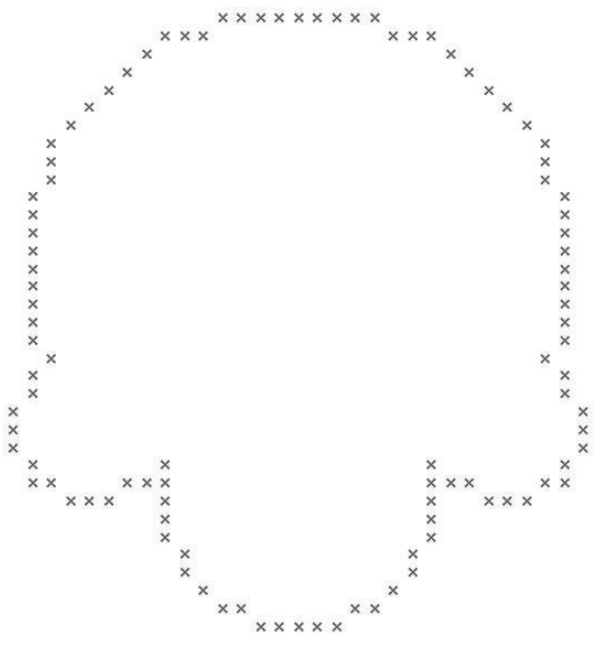

(a) The point number $=102$

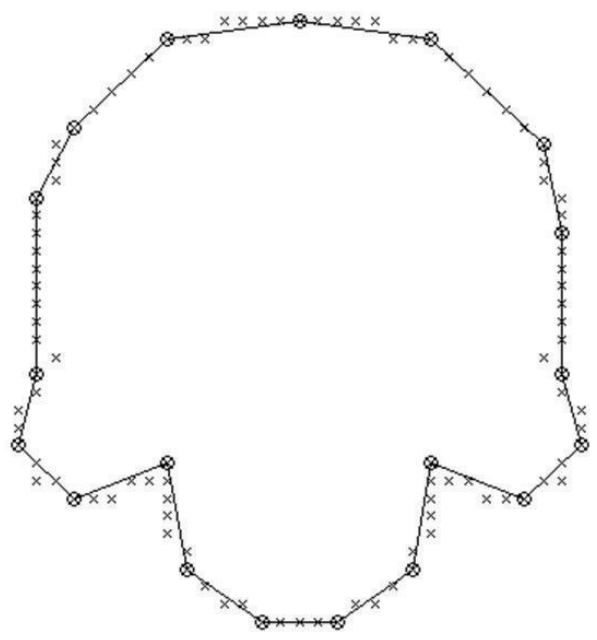

(c) Number of breakpoints $=19$

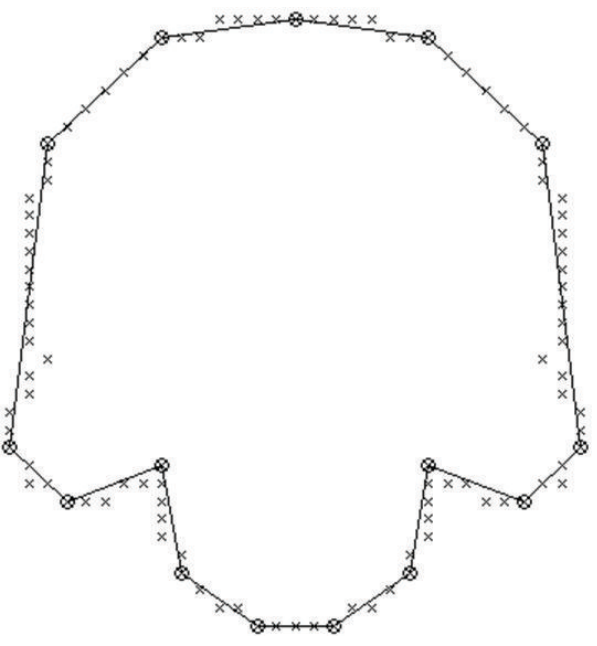

(b) Number of breakpoints $=15$

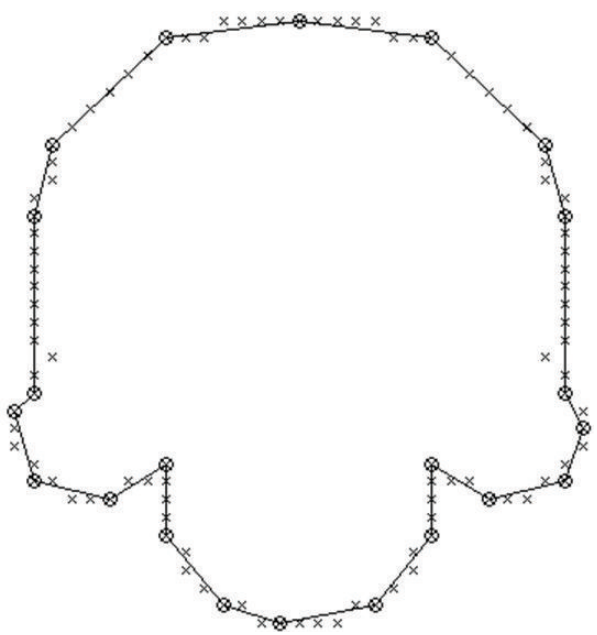

(d) Number of breakpoints $=22$

FIGURE 5: The approximated polygons for the semicircle-shaped curve obtained by the present method. (a) Original curve and (b)-(d) results using the present method.

TABLE 2: Experimental results for the chromosome-shaped curve.

\begin{tabular}{lccccc}
\hline \multirow{2}{*}{\begin{tabular}{l} 
Number of breakpoints \\
\cline { 2 - 4 }
\end{tabular}} & ISE (best) & DE-based method [8] & \multicolumn{3}{c}{ Present method } \\
ISE (average) & S.D. & ISE (best) & ISE (average) & 23.730 \\
12 & 23.730 & 23.730 & 0.000 & 23.730 & 0.000 \\
15 & 6.403 & 6.905 & 0.342 & 5.818 & 5.818 \\
\hline
\end{tabular}

TABLE 3: Experimental results for the leaf-shaped curve.

\begin{tabular}{|c|c|c|c|c|c|c|}
\hline \multirow{2}{*}{ Number of breakpoints } & \multicolumn{3}{|c|}{ DE-based method [8] } & \multicolumn{3}{|c|}{ Present method } \\
\hline & ISE (best) & ISE (average) & S.D. & ISE (best) & ISE (average) & S.D. \\
\hline 12 & 80.834 & 103.944 & 11.751 & 84.519 & 86.236 & 1.339 \\
\hline 17 & 42.400 & 47.354 & 4.699 & 21.314 & 26.219 & 2.685 \\
\hline 19 & 30.862 & 36.158 & 3.281 & 15.331 & 15.909 & 0.336 \\
\hline
\end{tabular}




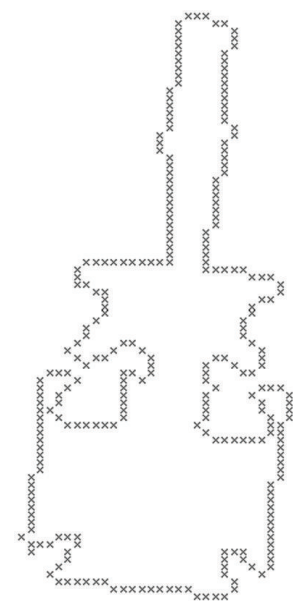

(a) The point number $=$ 267

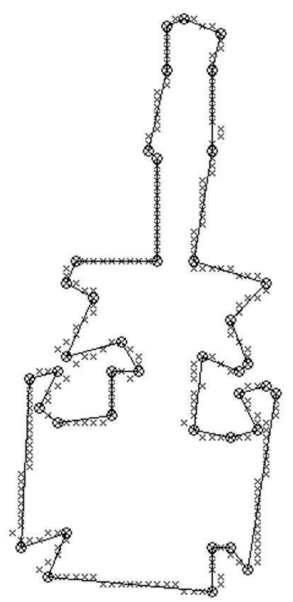

(b) Number of breakpoints $=40$

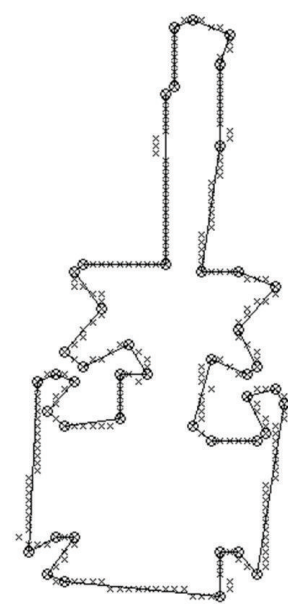

(c) Number of breakpoints $=45$

FIGURE 6: The approximated polygons for the screwdriver-shaped curve obtained by the present method. (a) Original curve and (b)-(c) results using the present method.

TABLE 4: Experimental results for the semicircle-shaped curve.

\begin{tabular}{|c|c|c|c|c|c|c|}
\hline \multirow{2}{*}{ Number of breakpoints } & \multicolumn{3}{|c|}{ DE-based method [8] } & \multicolumn{3}{|c|}{ Present method } \\
\hline & ISE (best) & ISE (average) & S.D. & ISE (best) & ISE (average) & S.D. \\
\hline 15 & 18.531 & 20.731 & 0.840 & 14.399 & 14.399 & 0.000 \\
\hline 19 & 12.519 & 13.377 & 0.543 & 10.115 & 10.439 & 0.217 \\
\hline 22 & 9.542 & 10.023 & 0.594 & 7.129 & 7.393 & 0.096 \\
\hline
\end{tabular}

minimum integral square error. Consequently, the NABC algorithm is a new population-based method and it has the advantages of finding the global or near-global minimum of a multimodal search space, having fast convergence, being simple and flexible, and using few control parameters. The present method simulates the behavior of honey bees foraging to develop the algorithm to select adequate breakpoints for polygonal approximation.

The method has been tested with four different wellknown curves. The present method is efficient in dealing with the application because the processing time of all cases is less than 1 second. Compared with the DE-based method, the present method can obtain better approximation results in most cases for both average solutions and best solutions. For the screwdriver-shaped curve and the fishshaped curve, the present method can also obtain good approximation results. From these experiments, these results have demonstrated the feasibility of the present method for polygonal approximation of digital curves with line segments. 


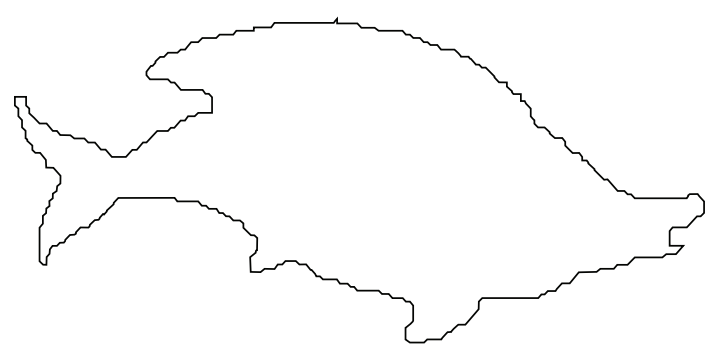

(a) The point number $=537$

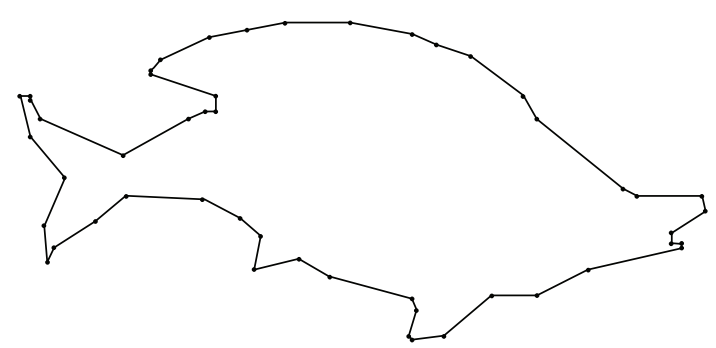

(b) Number of breakpoints $=50$

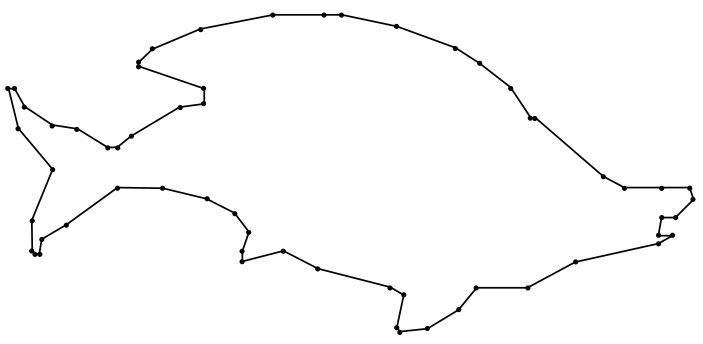

(c) Number of breakpoints $=60$

FIGURE 7: The approximated polygons for the fish-shaped curve obtained by the present method. (a) Original curve and (b)-(c) results using the present method.

TABLE 5: Processing time for finding various approximation results.

\begin{tabular}{lcc}
\hline Approximation & Number of breakpoints & Time (s) \\
\hline Figure 2(b) & 6 & 0.216 \\
Figure 2(c) & 11 & 0.262 \\
Figure 2(d) & 13 & 0.272 \\
Figure 3(b) & 6 & 0.273 \\
Figure 3(c) & 12 & 0.319 \\
Figure 3(d) & 15 & 0.346 \\
Figure 4(b) & 12 & 0.544 \\
Figure 4(c) & 17 & 0.581 \\
Figure 4(d) & 19 & 0.609 \\
Figure 5(b) & 15 & 0.488 \\
Figure 5(c) & 19 & 0.525 \\
Figure 5(d) & 22 & 0.562 \\
\hline
\end{tabular}

TABLE 6: Experimental results for the screwdriver-shaped curve and the fish-shaped curve.

\begin{tabular}{lcccc}
\hline Curve & $\begin{array}{c}\text { Number of } \\
\text { breakpoints }\end{array}$ & $\begin{array}{c}\text { ISE } \\
\text { (best) }\end{array}$ & $\begin{array}{c}\text { ISE } \\
\text { (average) }\end{array}$ & S.D. \\
\hline Screwdriver-shaped & 40 & 44.489 & 47.636 & 1.562 \\
curve & 45 & 35.475 & 38.240 & 1.786 \\
\hline \multirow{2}{*}{ Fish-shaped curve } & 50 & 88.722 & 93.215 & 2.657 \\
& 60 & 72.864 & 76.574 & 2.323 \\
\hline
\end{tabular}

\section{Conflict of Interests}

The author declares that there is no conflict of interests regarding the publication of this paper.

\section{References}

[1] R. C. Gonzalez and R. D. Woods, Digital Image Processing, Addison-Wesley, Reading, Mass, USA, 1992.

[2] C.-H. Teh and R. T. Chin, "On the detection of dominant points on digital curves," IEEE Transactions on Pattern Analysis and Machine Intelligence, vol. 11, no. 8, pp. 859-872, 1989.

[3] B. K. Ray and K. S. Ray, "Determination of optimal polygon from digital curve using $\mathrm{L}_{1}$ norm," Pattern Recognition Letters, vol. 26, no. 4, pp. 505-509, 1993.

[4] S.-C. Pei and J.-H. Horng, "Optimum approximation of digital planar curves using circular arcs," Pattern Recognition, vol. 29, no. 3, pp. 383-388, 1996.

[5] S.-C. Huang and Y.-N. Sun, "Polygonal approximation using genetic algorithms," Pattern Recognition, vol. 32, no. 8, pp. 1409$1420,1999$.

[6] S.-Y. Ho and Y.-C. Chen, "An efficient evolutionary algorithm for accurate polygonal approximation," Pattern Recognition, vol. 34, no. 12, pp. 2305-2317, 2001.

[7] P.-Y. Yin, "Ant colony search algorithms for optimal polygonal approximation of plane curves," Pattern Recognition, vol. 36, no. 8, pp. 1783-1797, 2003.

[8] B. Sarkar, "An efficient method for near-optimal polygonal approximation based on differential evolution," International Journal of Pattern Recognition and Artificial Intelligence, vol. 22, no. 6, pp. 1267-1281, 2008.

[9] A. Kolesnikov, "ISE-bounded polygonal approximation of digital curves," Pattern Recognition Letters, vol. 33, no. 10, pp. 13291337, 2012.

[10] B. Wang, D. Brown, X. Zhang, H. Li, Y. Gao, and J. Cao, "Polygonal approximation using integer particle swarm optimization," Information Sciences, vol. 278, pp. 311-326, 2014.

[11] Y. Xu, P. Fan, and L. Yuan, "A simple and efficient artificial bee colony algorithm," Mathematical Problems in Engineering, vol. 2013, Article ID 526315, 9 pages, 2013. 
[12] D. Karaboga and B. Basturk, "A powerful and efficient algorithm for numerical function optimization: artificial bee colony (ABC) algorithm," Journal of Global optimization, vol. 39, no. 3, pp. 459-471, 2007.

[13] D. Karaboga and B. Basturk, "On the performance of artificial bee colony (ABC) algorithm," Applied Soft Computing Journal, vol. 8, no. 1, pp. 687-697, 2008.

[14] D. Karaboga and B. Akay, "A comparative study of artificial Bee colony algorithm," Applied Mathematics and Computation, vol. 214, no. 1, pp. 108-132, 2009.

[15] D. Karaboga and C. Ozturk, "Neural networks training by artificial bee colony algorithm on pattern classification," Neural Network World, vol. 19, no. 3, pp. 279-292, 2009.

[16] C. Xu and H. Duan, "Artificial bee colony (ABC) optimized edge potential function (EPF) approach to target recognition for lowaltitude aircraft," Pattern Recognition Letters, vol. 31, no. 13, pp. 1759-1772, 2010.

[17] C. Zhang, D. Ouyang, and J. Ning, "An artificial bee colony approach for clustering," Expert Systems with Applications, vol. 37, no. 7, pp. 4761-4767, 2010.

[18] G. Zhu and S. Kwong, "Gbest-guided artificial bee colony algorithm for numerical function optimization," Applied Mathematics and Computation, vol. 217, no. 7, pp. 3166-3173, 2010.

[19] Q.-K. Pan, M. F. Tasgetiren, P. N. Suganthan, and T. J. Chua, "A discrete artificial bee colony algorithm for the lot-streaming flow shop scheduling problem," Information Sciences, vol. 181, no. 12, pp. 2455-2468, 2011.

[20] M.-H. Horng, "Multilevel thresholding selection based on the artificial bee colony algorithm for image segmentation," Expert Systems with Applications, vol. 38, no. 11, pp. 13785-13791, 2011.

[21] W. Gao, S. Liu, and L. Huang, "A global best artificial bee colony algorithm for global optimization," Journal of Computational and Applied Mathematics, vol. 236, no. 11, pp. 2741-2753, 2012.

[22] A. Draa and A. Bouaziz, "An artificial bee colony algorithm for image contrast enhancement," Swarm and Evolutionary Computation, vol. 16, pp. 69-84, 2014.

[23] S. Talatahari, H. Mohaggeg, K. Najafi, and A. Manafzadeh, "Solving parameter identification of nonlinear problems by artificial bee colony algorithm," Mathematical Problems in Engineering, vol. 2014, Article ID 479197, 6 pages, 2014.

[24] S. Sarkar, D. Roy, and R. M. Vasu, "A perturbed martingale approach to global optimization," Physics Letters A, vol. 378, no. 38-39, pp. 2831-2844, 2014. 


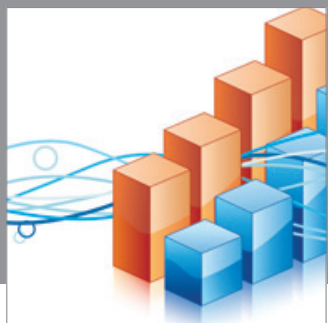

Advances in

Operations Research

mansans

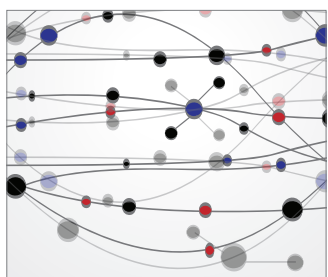

The Scientific World Journal
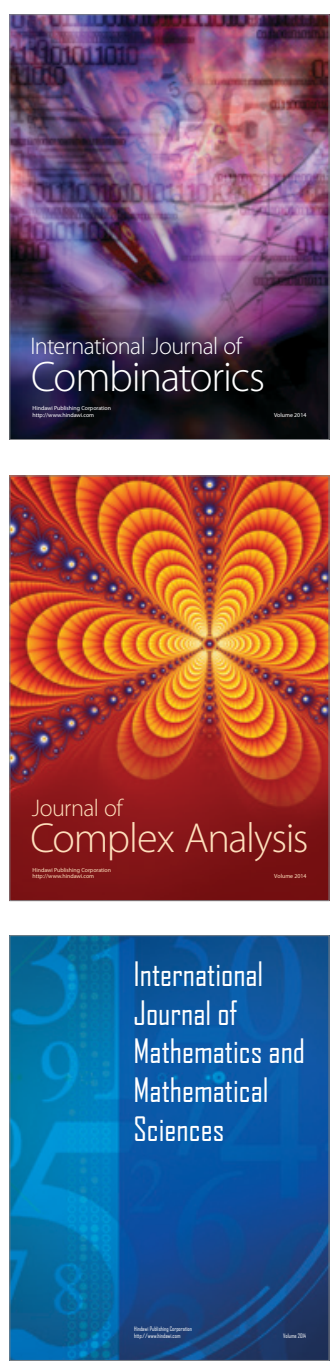
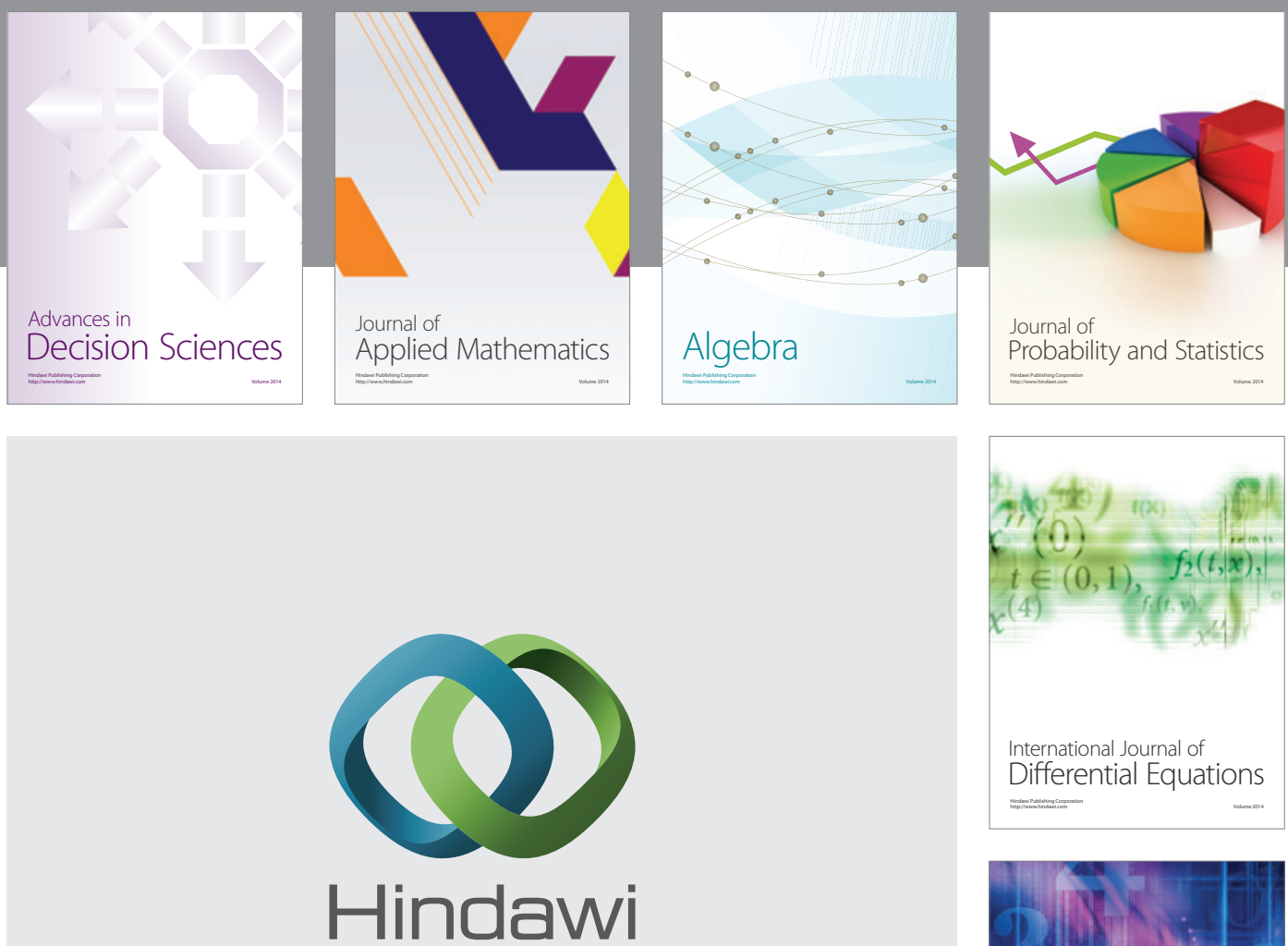

Submit your manuscripts at http://www.hindawi.com
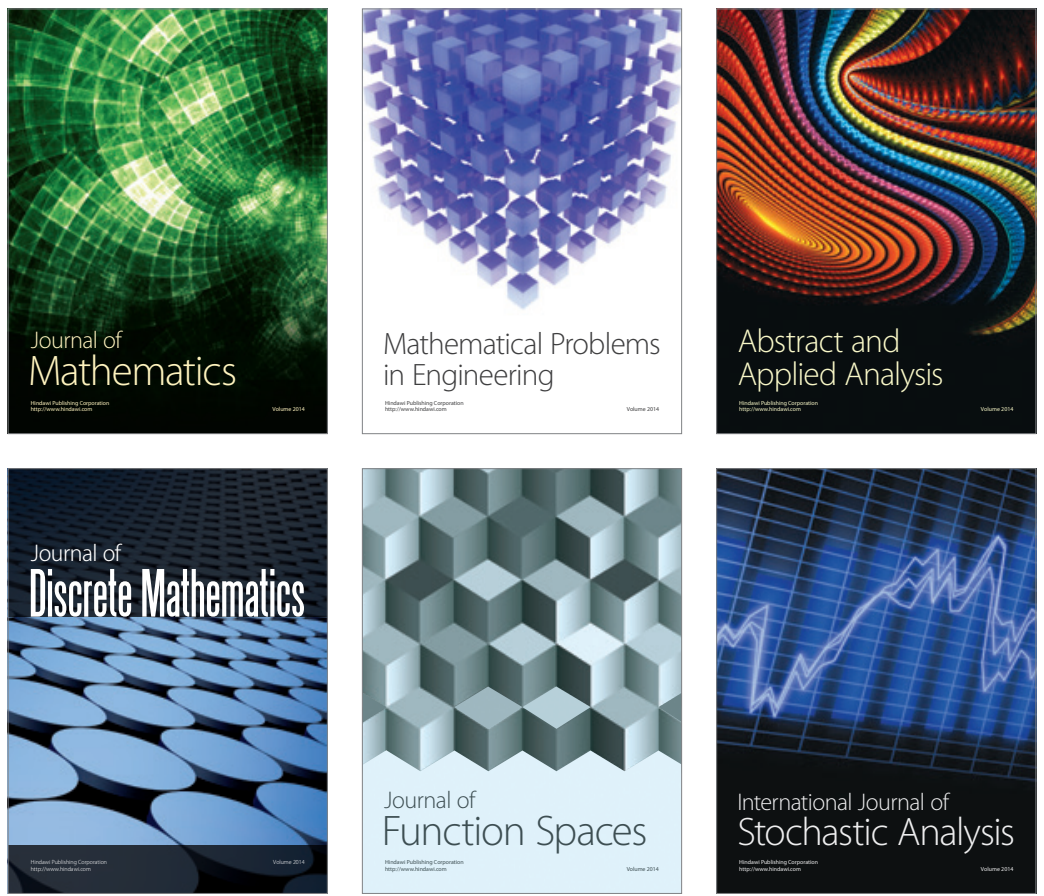

Journal of

Function Spaces

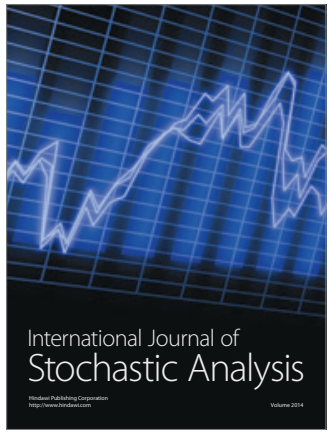

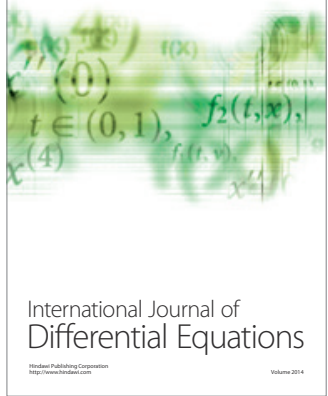
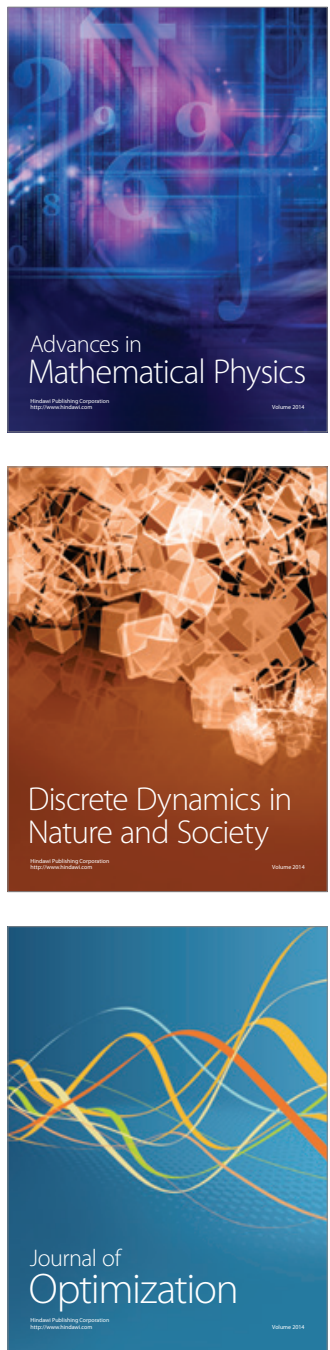